\author{
Katharina Glatz-Krieger · Udo Spornitz • Alain Spatz • \\ Michael J. Mihatsch · Dieter Glatz
}

\title{
Factors to keep in mind when introducing virtual microscopy
}

Received: 17 June 2005 / Accepted: 4 October 2005 / Published online: 22 November 2005

(C) Springer-Verlag 2005

\begin{abstract}
Digitization of glass slides and delivery of socalled virtual slides (VS) emulating a real microscope over the Internet have become reality due to recent improvements in technology. We have implemented a virtual microscope for instruction of medical students and for continuing medical education. Up to 30,000 images per slide are captured using a microscope with an automated stage. The images are post-processed and then served by a plain hypertext transfer protocol (http)-server. A virtual slide client (vMic) based on Macromedia's Flash MX, a highly accepted technology available on every modern Web browser, has been developed. All necessary virtual slide parameters are stored in an XML file together with the
\end{abstract}

Universal resource locators (URLs) visited at the time of writing (June 2005) may change or vanish with lapse of time. Therefore and because of usability, we have created a web page with all URLs that will be updated on a regular basis: http://kathrin.unibas.ch/ $\mathrm{pub} / 2005-06$.

K. Glatz-Krieger · M. J. Mihatsch

Institute for Pathology,

University Hospital of Basel,

Basel, Switzerland

\section{U. Spornitz}

Anatomical Institute,

University of Basel,

Basel, Switzerland

\section{A. Spatz}

Institute Gustave Roussy,

Villejuif, France

D. Glatz

IT Department,

University of Basel,

Basel, Switzerland

K. Glatz-Krieger $(\square)$

Institut für Pathologie,

Schönbeinstrasse 40

CH-4031 Basel, Switzerland

e-mail: Katharina.Glatz@unibas.ch

Tel.: +41-61-2652880 image. Evaluation of the courses by questionnaire indicated that most students and many but not all pathologists regard virtual slides as an adequate replacement for traditional slides. All our virtual slides are publicly accessible over the World Wide Web (WWW) at http://vmic.unibas.ch. Recently, several commercially available virtual slide acquisition systems (VSAS) have been developed that use various technologies to acquire and distribute virtual slides. These systems differ in speed, image quality, compatibility, viewer functionalities and price. This paper gives an overview of the factors to keep in mind when introducing virtual microscopy.

Keywords Telepathology · Virtual slides ·

Virtual microscopy $\cdot$ Continuing medical education

Abbreviations NA: Numerical aperture $\cdot V M$ : Virtual microscope · VS: Virtual slide · VSAS: Virtual slide acquisition system - VSS: Virtual slide system - WWW: World Wide Web

\section{Introduction}

Only recently, advances in computer technology have been made that allow to digitize a complete glass slide. Such virtual slides (VS) can be accessed over the World Wide Web (WWW), making them available to a large audience. Since 1998, several virtual slide systems (VSS) have been developed by different commercial and academic bodies (http://www.pathmax.com/apiii/exp.html, http://141.214.6. 12/cyberscope631) $[1-4,6,10,15,18,20,21,23,24,26]$. The value of these virtual microscopes varies considerably depending on the technology used for image acquisition, processing, delivery and viewer functionality. Only few sites are publicly available over the Internet (http://interpath1.uio. no/telemedisin/WebInterPath/interpathindex.htm, http://www. bacuslabs.com, http://virtualmicroscope.osu.edu/virtual. htm, http://neuroinformatica.com, http://images.scanscope. com, http://www.telepathology.dcu.ie/vps02.php3?hits=, http://www2.primed.helsinki.fi/webmicroscope/atlases/ breast/brcatlas_start.asp). An outstanding collection of 
more than 900 VS is accessible for public use and education from the University of Iowa (http://www.path.uiowa.edu/ virtualslidebox).

There is no generally accepted definition of the term "virtual microscopy". In the past, this term has variably been used for sending single images to a colleague by e-mail, for online atlases containing large single images or for image collections showing various sectors of a slide at different magnifications. Only recently, it became possible to digitize entire slides at high magnification, allowing exploration of the tissue on a computer screen in a manner analogous to a real microscope, thus justifying the designation "virtual microscope". We advocate the restricted use of the term "virtual slide" for completely digitized slides at full resolution (scanned at $20 \times$ or higher), allowing the pathologist to make a clear-cut diagnosis. Similarly, a virtual microscope should provide at least the functionality of a real microscope (scrolling and zooming of the VS) and may offer additional functionalities like Web accessibility, annotations or automated image analysis.

We have developed a VSS called vMic for public WWW access (http://vmic.unibas.ch). Emphasis was laid on open standards and maximal image quality still allowing for reasonable download times. In an earlier paper, we discussed the theoretical aspects of virtual microscopy [9]. We now report on the first successful applications of vMic in teaching. The parameters to be considered when planning the implementation/acquisition of a VSS are discussed extensively.

\section{Construction of the virtual slide system}

We have built a high-quality/low-cost VSS based on the theoretical requirements described in detail earlier [9]. In short, a typical setup for a VSS consists of a virtual slide acquisition system (VSAS), a server that delivers the VS via Internet/WWW to the user and a viewer - preferably a Web-based version.

The demands for our VSAS were:

- Highest possible image quality

- Use of standard components available on the market

- Least possible effort for custom developments (software)

- Highest possible reusability of the investment

Our VSAS consists of commercially available components (Table 1) and includes: a microscope with a robotic stage, high-quality objectives, and a camera for slide acquisition. An acquisition software moves the stage and acquires the images. Due to limitations of the mechanical precision of components (motorized stage, camera mounting, reproducibilitiy, hysteresis, thermal drift, different $z$ positions due to autofocus), the creation of a nearly seamless VS is very tedious when using a standard robotic microscope [23]. In order to circumvent time-consuming calibrations, overlaps of about $3-5 \mu \mathrm{m}$ at $40 \times$ magnification at the intersection of two individual images were deliberately chosen for our VSAS. For viewing the digitized slides in a Web browser, a Flash application
Table 1 Components of the vMic slide acquisition system

Microscope Zeiss Axioskop 2 mot plus

Objectives Zeiss Plan Apochromat $40 \times / 0.95$ for scanning the slides, Zeiss Neofluar 2.5 for overviews

Motorized Maerzhaeuser stage SCAN 75*50

stage

Stage Ludl Controller MAC5000

controller

Camera Zeiss AxioCam HRc

Software Zeiss KS400 (includes a macro language enabling to programme a VSAS)

Computers 2 computers with $\mathrm{P} 4 / 2.4 \mathrm{GHz}$; one as the capturing system, the second for post-processing

was developed that allows the examination of the slides at different magnifications and to move continuously within a slide, closely emulating the functionality of a real microscope. In addition, the user may directly jump to regions of interest guided by an overview image with a cross and a rectangle indicating the current viewing position (Fig. 1). Annotations describe regions of interest and link to extra information on the Web.

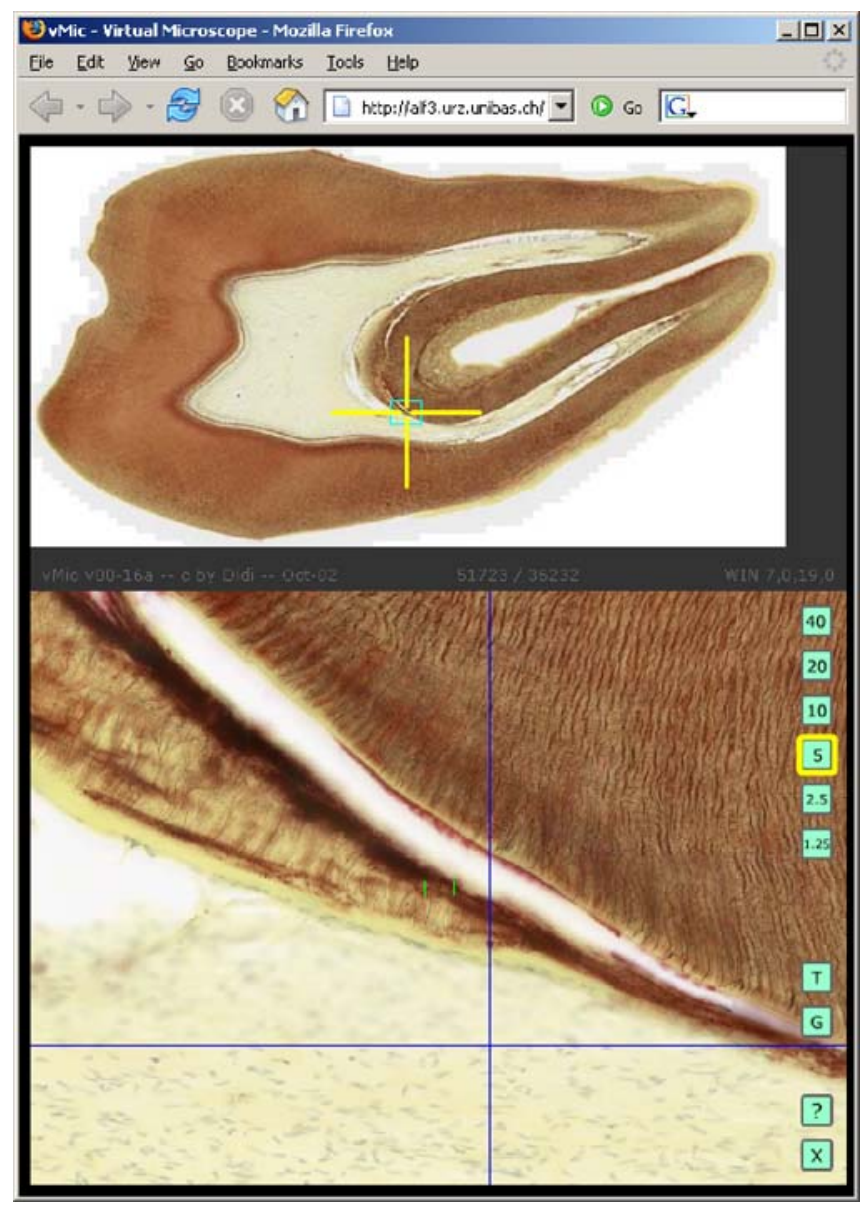

Fig. 1 Virtual slide of the Oral Histology course: The upper part of the vMic viewer shows an overview of a molar tooth. A blue frame plus yellow crosshairs indicate the field of view that is shown at $5 \times$ magnification in the lower part 
The optical parameters of the VSAS have been chosen to match the limitation of the optical resolution [9]. Maximal image quality was achieved at the cost of low acquisition speed. Each single image was autofocussed, and the AxioCam was set to scan mode at $1,300 * 1,030$ pixel, providing full colour information for each pixel.

To reduce capturing time, a mask representing the region of interest was predefined to guide the roboter.

The captured images were post-processed with Javascripted Adobe Photoshop. This step included correction of colour and gradation as well as applying an unsharp mask. The latter was only applied to the lightness channel in Lab space, generating crisper images without introducing unwanted colour artefacts. Details of post-processing are illustrated at http://kathrin.unibas.ch/pub/2005-06. Finally, a multi-resolution stack was produced and transferred to the Web server. The viewer (vMic, http://vmic.unibas.ch) and the server have been described in detail previously [9]. Capturing as well as post-processing may last for several hours depending on the slide size and the number of images taken. The components of the described system adhere to open standards and are interchangeable with further developments in the field.

\section{vMIC applications in teaching}

After its introduction, vMic (http://vmic.unibas.ch) has primarily been used for teaching purposes: as a component of an online histopathology course for medical students (http://alf3.urz.unibas.ch/hipaku), in a "virtual histology" laboratory for students in dentistry (http://oralhisto.unibas. ch) and in three slide seminars for surgical pathologists (http://vmic.unibas.ch/patho/seminar). The slide seminars had all been organized by sections of the International Academy of Pathology.

\section{Histology course for students in dentistry}

Students in dentistry have traditionally been taught dental histology by means of introductory lectures and practical hands-on microscopy courses. A professor of anatomy taught histology showing microscopic slides on a microscope projected with a video projector. Every student needed a microscope and a set of glass slides for the laboratory sessions. Many of these valuable glass slides had already been lost, broken, or faded because of prolonged storage and recurrent intensive exposure to the microscopic illumination. Some of the slides used in this course were very old and could not be replaced due to lack of technicians trained in the various technologies required for the preparation of dental tissues. Furthermore, the procedures applied in the production of some of the slides, e.g. grinding of enamel, is very time consuming, and human resources are scarce. In order to avoid further loss of valuable material, a selection of the best slides used during the course was digitized and the course was moved from the microscopy lab to a computer room. Every student now has identical material of optimal quality to study on a monitor, and the instructor can indicate the coordinates of the area under discussion. Prior to examinations, students may review the slides at home or at one of the numerous public computer workstations offered by the university. Individual use of the course is alleviated by an information sheet provided as a "PDF" file that can be downloaded. The new course (http://oralhisto.unibas.ch) was held for the first time during the winter semester 2003/2004. On average, $85 \%(29 / 34)$ of the students in dentistry participated in each virtual microscopy session. Overall, the students were highly satisfied with the new teaching method. The course had been evaluated with an online questionnaire after the last virtual microscope session (Table 2). Remarkably, the students rated image quality of vMic equal $(6 \times)$ or even superior $(7 \times)$ to the image quality of their traditional microscopes, whereas no student indicated the reverse. Last but not least, the grades for the histology exams went up from an average of 5.1 to 5.3 (6 being the highest grade). This, however, might be due to various not course-related factors and certainly needs further evaluation.

Histology course for medical students

We have included some of the more than $900 \mathrm{VS}$ of the virtual slidebox of histopathology (http://www.path.uiowa. edu/virtualslidebox) and few vMic slides in our Web-based histopathology course for medical students (http://alf3.urz. unibas.ch/hipaku).

Traditional slide seminar with additional virtual slides

For the course "Diagnosis of Melanocytic Lesions: Common Problems for the Practicing Pathologist" of the French section of the International Academy of Pathology, VS had been produced in addition to traditional slides. The two courses held in Paris in December 2003 and in November 2004 had been organized by R.L. Barnhill, M.G. Cook, D. J. Ruiter and A. Spatz. The main topic of this annual course is melanocytic lesions that are known for their highly variable appearance in different step sections. Therefore, it was in many cases impossible to provide a set of glass slides for each of the around 80 to 100 participants showing all the diagnostic details of the 12 cases discussed during the 1-day course meeting. In order to circumvent this problem, a representative step section of each course slide was digitized and made available for viewing via the Internet of all the participants prior to the course meeting (http://vmic.unibas.ch/patho/seminar).

Traditional slide seminar with virtual slides (without glass slides)

Transplant pathology was the main topic of the 88th annual meeting of the German Society of Pathology in Rostock in June 2004. On this occasion, the German section of the 
Table 2 Evaluation of oral histology course and slide seminars (Paris 2003/04 and Rostock 2004) by participants

\begin{tabular}{|c|c|c|c|}
\hline & Oral Histology students & Paris Dermatopathology & Rostock Renal Pathology \\
\hline \multicolumn{4}{|c|}{$\begin{array}{l}\text { The download time for the individual image segments is... (The type of internet connection used by the respondents is indicated in brackets. } \\
\text { All students had a LAN connection.) }\end{array}$} \\
\hline Fast & 3 & $2(2 \mathrm{~T} 1)$ & $2(2 ?)$ \\
\hline $\mathrm{OK}$ & 9 & 9 (2 ISDN, 5 ADSL, 2 ?) & 7 (4 T1, 2 ?, 1 ISDN) \\
\hline Slow & 1 & 13 (4 ISDN, 3 ADSL, 6 ?) & 2 (1 ISDN, 1 Modem) \\
\hline Inacceptably slow & 0 & 3 (1 modem, 1 ADSL, 1 ISDN) & 0 \\
\hline \multicolumn{4}{|c|}{ Making a diagnosis on a VS compared to a traditional slide is... } \\
\hline Easier & 3 & 0 & 0 \\
\hline Comparable & 9 & 6 & 4 \\
\hline More difficult & 1 & 17 & 7 \\
\hline Impossible & 0 & 2 & 0 \\
\hline \multicolumn{4}{|c|}{ Would you like VS seminars in addition to the conventional seminars? } \\
\hline Yes & - & 20 & 8 \\
\hline No & - & 6 & 3 \\
\hline \multicolumn{4}{|l|}{ For future conventional slide seminars, I would prefer... } \\
\hline VS as a supplement & - & 18 & 7 \\
\hline VS only & - & 4 & 4 \\
\hline Traditional slides only & - & 2 & 0 \\
\hline \multicolumn{4}{|c|}{ For what purposes would you like to use a virtual microscope? (all that apply) } \\
\hline Slide seminars & - & 26 & 8 \\
\hline Other kinds of online education & - & 25 & 9 \\
\hline Online consultation (second opinion) as consulter & - & 12 & 7 \\
\hline Online consultation (second opinion) as expert & - & 3 & 5 \\
\hline I will not use virtual microscopy & - & 4 & 0 \\
\hline
\end{tabular}

Some participants of slide seminars did not answer all of the questions. Therefore, the number of respondents for each question varies

International Academy of Pathology organized a slide seminar exclusively with VS. As needle biopsies of the kidney are very small, slide seminars with many participants on this topic were not feasible to date owing to lack of sufficient tissue. The six seminar cases represented various conditions of kidney transplant pathology. The slides were contributed by three specialists in the field-M.J. Mihatsch, H. Regele, and H.-J. Gröne-who discussed their cases on the occasion of the course meeting in Rostock. The slides were published online 1 month prior to the course meeting. The participants could either view the cases at home or at a computer station in Rostock.

The participants of the slide seminars were asked to answer a questionnaire about vMic (Table 2). While most respondents expressed positive opinions about the virtual microscope ("it is the future"), others were more sceptic ("probably useful for slide seminars but not yet good enough for consultation"). The image quality of the VS was rated as either excellent $(62 \%)$ or good $(38 \%)$. Nobody had voted for moderate or poor.

All slide seminars are publicly accessible (http://vmic. unibas.ch/patho/seminar).

\section{Added value of virtual slides}

VS should not simply be regarded as a mere replacement of traditional glass slides. This new technology offers several advantages and applications to pathology that could not be provided before $[3-5,7,8,10,12,15,16,18-22,24,26]$. The overview image facilitates orientation within a given section (Fig. 1). Annotations that lead the user to a specific location within the slide are a valuable tool not only in the teaching setting but also for consultations. The one-time digitization of the histological glass slides for teaching purposes is much less time consuming and expensive than the production of a traditional slide set that additionally comprises the replacement of lost, broken or faded sections and delivery. The decision about which cases are to be presented in a course no longer depends on the availability of a large amount of representative tissue but exclusively on didactics. Cytopathology courses $[7,19,27]$ or slide seminars in subspecialties traditionally relying on small biopsy samples or needle biopsies such as haematopathology, dermatopathology (http://vmic.unibas.ch/patho/seminar, http://www.pathmax. com/apiii/exp.html), hepatopathology or nephropathology (http://vmic.unibas.ch/patho/seminar) can now be offered to a large audience. For digitization, a step section representative of the lesion and of best quality is chosen. The fact that the best possible identical step section of a given lesion is allocated to all users is not only desirable for teaching purposes but represents an optimal condition for consensus conferences in research settings [26] or measures of quality control $[7,17]$. A group of experts from any part of the world can use the "virtual multi-header microscope" to examine a slide together via the WWW. 


\section{Consumer needs}

If virtual microscopy is to be widely accepted by pathologists for diagnostic purposes, image quality and functionality should be comparable to that of a traditional microscope. The acceptance of Web-based technology is generally very high in the student population. The improvement of technology should be paralleled by increasing computer competency of the upcoming generation of pathologists $[11,14]$. Students have already been shown to rate the quality of images and navigation of a virtual microscope equal to or even better than a real microscope $[2,10,18]$. While real slides need adjustments of focus, lighting and condensor, VS are always ready to use. In contrast, most pathologists found it more difficult to make a diagnosis on vMic slides than with traditional slides. This is probably due in part to unfamiliarity with this new technology and in part to a rather small display and delayed response time when using slow Internet connections.

When we developed vMic, we decided to obtain the highest possible image quality accepting increased time requirements for image acquisition and download. It has to be taken into account that rating of image quality not only depends on the virtual microscope but also on the quality of the computer screen of the user and the quality of the physical microscope vMic is compared to. Due to limited budgets for medical education, microscopes in student labs normally do not feature best-of-breed optics to minimize costs, while VS can be maximized for quality. The latter probably explains why more than half of students in dentistry rated vMic images as superior to a real microscope image.

The overall quality of a VSS will not only be measured by its visual quality and its ability to perfectly emulate a traditional microscope but will also depend on additional functionalities like annotations, automatized slide analysis or discussion tools.

\section{Considerations for acquisition/implementation of a virtual slide system}

Nowadays, many companies offer VSAS or even complete VSS (http://www.soft-imaging.de/rd/english/3412.htm, http:// www.telepathologycity.com/systems.htm). They foster different technological approaches, and the price tag varies by a factor of 6 and more. The following discussion of the most relevant parameters in virtual microscopy is intended to help institutions that plan to establish their own VSS.

\section{Acquisition speed}

One obvious and central weakness of most existing VSAS is acquisition speed. As long as the system is only planned to serve educational purposes (e.g. vMic, http://vmic. unibas.ch), this shortcoming may be acceptable. A single scan may last for hours. However, if VS are to be intro- duced in routine diagnostics, a very fast and automated system - mostly at a higher price - is mandatory.

Four systems that meet the latter conditions are (incomplete list of available products):

- DMetrix DX-40 scanner (first systems delivered in 2004; http://www.dmetrix.net)

- Olympus .Slide (pronounced dot-slide; available since beginning of 2005; http://www.soft-imaging.de/rd/ english $/ 3412 . \mathrm{htm})$

- Aperio ScanScope (available since more than 1 year ago; http://images.scanscope.com)

- InterScope Xcellerator (http://www.interscopetech.com)

The most promising technology is the one used in the DX-40 scanner from DMetrix. This VSAS consists of an array of multiple miniaturized microscopes allowing for ultra-rapid digitization of a whole slide in less than a minute [26].

$20 \times$ vs $40 \times$ objective

In traditional settings, $40 \times$ objectives with a numerical aperture (NA) of 0.75 are used for diagnostic purposes. A 40× Plan Apochromat objective (normally used to take images) has up to $\mathrm{NA}=0.95$, providing higher lateral resolution, while depth of sharpness is reduced. A $20 \times$ Plan Apochromat objective with $\mathrm{NA}=0.75$ results in the same lateral resolution as a standard $40 \times$ objective used for diagnostics.

Some suppliers therefore argue that by using a $20 \times$ Plan Apochromat instead of a $40 \times$ objective, the scanning time would be reduced by a factor of 4 without cutting on image quality and resolution of the resulting VS. Yet, that claim is only true when comparing two objectives with the same NA. A $40 \times / \mathrm{NA}=0.95$ clearly provides higher lateral resolution. Furthermore, the resolution of the camera must be considered as commented on in the next section.

\section{Video camera vs still camera and camera resolution}

Video cameras are in general faster than still cameras. They can grab up to 30 images per second, whereas still cameras take up to $1 \mathrm{sec}$ per image. On the other hand, still cameras deliver up to 12 to 20 megapixels per image, whereas video cameras are limited to about 0.3 to 1.3 megapixels per image $(640 * 480$ to $1,300 * 1,030)$.

Dedicated VS scanners may also use specialized sensors (Aperio, http://images.scanscope.com; DMetrix, http:// www.dmetrix.net).

For optimal colour information, the red, green and blue (RGB) component of light should be collected at each pixel. Many charge coupled device (CCD)-chips use a socalled Bayer mask instead (Fig. 2), which reduces colour information significantly.

Three types of technology are available to gather colour information for every single pixel: (a) three-chip cameras- 


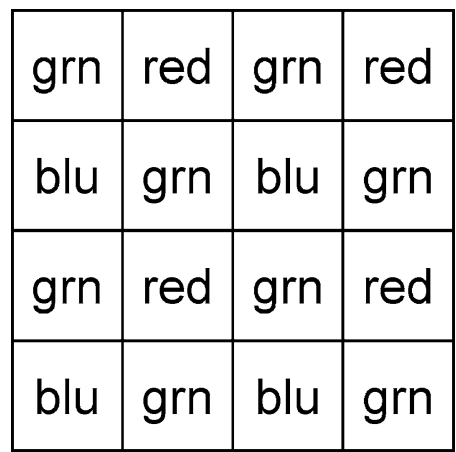

Fig. 2 Bayer mask. Chequer-board-like filter for RGB (red, green, blue). The intensity of each pixel of the sample is measured at one wavelength (colour) only. Full colour information will then be interpolated, not really measured

the light path is split and RGB components are captured each on a separate chip, (b) scan mode - a sensor is equipped with a Bayer mask and the whole chip is piezoelectrically repositioned four times per image to measure each component separately (e.g. AxioCam HR; image comparison, (http://alf3. urz.unibas.ch/patho/pub/axiocam.pdf), (c) three-pass modethe sensor scans the sample in three successive scans each time using a different illumination for red, green and blue (e.g. DX-40, (http://www.dmetrix.net).

As a rule of thumb, approximately 1 to 1.5 megapixels are needed when taking pictures with a $40 \times$ objective and a standard camera to match the optophysical resolution of a standard microscope [9]. Increasing the resolution of the camera above this value increases image size without yielding more information and should therefore be avoided. When using a $20 \times$ Plan Apochromat objective for slide scanning, the camera resolution should be four times higher because the field of view is four times larger than with a $40 \times$ objective. Otherwise, image information is lost.

\section{Single/multiple focus planes and $z$-sharpening}

A major drawback of most VS is the lack of multiple focus planes that allow the pathologist and even more so the cytologist to slightly change focus from the main focus plain within the $z$-axis. Therefore, information contained within a real slide is lost in the VS due to a reduced depth of sharpness. To avoid this drawback, two different approaches have been realized, both scanning the slide at multiple focus planes, but:

(a) Delivers the additional planes on request to the browser (e.g. Bacus Laboratories WebSlide, (http://www.bacus labs.com).

(b) $z$-sharpening: a software algorithm extracts the sharpest areas from the different focal planes and combines them to a synthesised image with a high depth of sharpness (e.g. Olympus. Slide, http://www.soft-imag ing.de/rd/english/3412.htm; DMetrix, http://www.dme trix.net - announced as a future feature).
Both approaches significantly increase total scanning time. Bacus' WebSlide more closely simulates a real microscope - at the cost of increased download time and a magnitude of storage.

It remains to be evaluated which of the two approaches will lead to more accurate diagnoses with comparative studies of the two methods.

Single large image file vs matrix with thousands of images

Scanning large samples results in dozens of gigabytes of raw data. Advances in computer technology are by and by overcoming the problems arising from handling single large image files. However, only few software tools are able to handle (analysis, post-processing) such huge image files yet. For Web delivery, special server processes are needed for single-file VS, whereas matrices of small files can be served using a standard http demon.

Dynamic downsizing vs pyramidal multi-resolution stack

Two different approaches are used to view VS at different magnifications. Some systems only store the highest resolution on disk. For viewing VS at smaller magnifications, the image is dynamically downsized. This might be appropriate for a single-user system. However, in a multiuser and multi-access system, which is true for most Web applications, dynamical downsizing raises unnecessary high demands to the server [3]. A pyramidal stack stores images at different resolutions [9]. Most VSS use a pyramidal approach. In the case of vMic, this requires less than $12 \%$ additional storage space.

Proprietary systems vs open standards

Unfortunately, many suppliers offer VSS that use proprietary image file formats and viewers. Some have adopted Zoomify's technology (http://www.zoomify.com/customers). Although this is also a proprietary image file format, a lowcost developer kit enables the development of own viewers optimized for teaching, research or diagnostics. For maximal compatibility, VSAS should be able to export VS as a matrix of single-image files in a standard format.

It would be advantageous if image acquisition was decoupled from image delivery. This would not only foster interchange of VS but also the independent development of value-adding viewer applications for education, research and diagnostics. Open standards in the delivery of VS are the precondition for the construction of a semantic web of pathology (http://www.w3.org/2001/sw, http://www.inf.fuberlin.de/inst/ag-nbi/research/swpatho/icwe04.pdf). 
Sufficient image quality vs best possible quality

Most VSAS are designed to reach reasonable diagnostic quality allowing the pathologist to make a reliable diagnosis [4, 16, 20, 26]. Depending on the application, this approach is absolutely adequate since it minimizes storage space, scanning time and expenditures for optics. vMic on the other hand aims at maximal quality that is reached when equalling the optophysical limitations of the microscope $(40 \times, \mathrm{NA}=0.95)$. With the advent of improved technologies, maximal quality will sooner or later be standard, although minimal diagnostic quality will still be good enough in the future.

Overlapping images vs seamless images

Producing seamless VS using standard microscope technologies demands tedious alignment procedures [23] or time-consuming software stitching.

Dedicated VSAS (DMetrix, http://www.dmetrix.net; Olympus, (http:/www.soft-imaging.de/rd/english/3412.htm; Aperio, http://images.scanscope.com) produce seamless VS. Our user surveys have shown that overlaps are accepted by students and pathologists and are not regarded as a drawback in the use of VS.

\section{Purpose of application}

If VS are to be used for educational purposes, a low-cost solution may do since scanning time is not critical and overlaps are accepted by the users. VS in daily diagnostics as introduced at the University of Arizona [26] require a high throughput instrument including many terabyte of disk space.

First test, then buy

Some suppliers do not like customers to test their systems before purchase. Some of the systems we have evaluated would never have been able to deliver a VS. Most of the times, additional implementing work is needed depending on the user preferences. Before investing in an expensive VS equipment, the intended uses should be worked out and the possibility to test the system should be demanded from the supplier.

\section{Outlook}

Due to additional functionalities that a physical microscope cannot provide, virtual microscopy is a promising new tool, offering a wide range of new applications in the fields of education, research, diagnosis and quality control (http:// www.bacuslabs.com, http://virtualmicroscope.osu.edu) [2, $4,7,10,13,15,18,20,24,26,27]$.
High-speed broadband Internet connections are expected to be available in most laboratories soon, allowing most pathologists and students to profit from publicly accessible sites.

Storing information contained in a single VS (one focal plane only) takes up to ten CD-ROMs or one DVD. Replacing physical slide archives by digital archives is therefore not reasonable at present but will probably be possible within the next decades.

Fast slide-processing time is essential if VS are to be widely used for routine diagnostic pathology including intra-operative frozen sections and second-opinion consultations. The advent of easy-to-handle ultra-rapid slide scanners [25] will ensure a fast propagation of this most recent application in telepathology.

By participating in VS seminars, every pathologist can make a first step towards mastery of this trend-setting technique. The use of vMic (http://vmic.unibas.ch) slides is free of charge and does not require registration, allowing everyone to gain first experiences with a new way of looking at histology slides.

Acknowledgements The authors thank Drs. Ray Barnhill, Martin Cook, Dirk Ruiter, Heinz Regele and Hermann-Josef Gröne for the contribution of cases for the VS seminars. This work has been supported by a grant of the University of Basel.

\section{References}

1. Afework A, Beynon MD, Bustamante F, Cho S, Demarzo A, Ferreira R, Miller R, Silberman M, Saltz J, Sussman A, Tsang H (1998) Digital dynamic telepathology - the virtual microscope. Proc AMIA Symp: 912-916

2. Blake CA, Lavoie HA, Millette CF (2003) Teaching medical histology at the University of South Carolina School of Medicine: transition to virtual slides and virtual microscopes. Anat Rec B New Anat 275:196-206

3. Catalyurek U, Beynon MD, Chang C, Kurc T, Sussman A, Saltz J (2003) The virtual microscope. IEEE Trans Inf Technol Biomed 7:230-248

4. Costello SS, Johnston DJ, Dervan PA, O'Shea DG (2003) Development and evaluation of the virtual pathology slide: a new tool in telepathology. J Med Internet Res 5:e11

5. Dee FR, Lehman JM, Consoer D, Leaven T, Cohen MB (2003) Implementation of virtual microscope slides in the annual pathobiology of cancer workshop laboratory. Hum Pathol 34:430-436

6. Demichelis F, Barbareschi M, Dalla Palma P, Forti S (2002) The virtual case: a new method to completely digitize cytological and histological slides. Virchows Arch 441:159-164

7. Demichelis F, Della Mea V, Forti S, Dalla Palma P, Beltrami CA (2002) Digital storage of glass slides for quality assurance in histopathology and cytopathology. J Telemed Telecare 8:138-142

8. Giltnane JM, Rimm DL (2004) Technology Insight: identification of biomarkers with tissue microarray technology. Nature Clinical Practice Oncology 1:104-111

9. Glatz-Krieger K, Glatz D, Mihatsch MJ (2003) Virtual slides: high-quality demand, physical limitations, and affordability. Hum Pathol 34:968-974

10. Harris T, Leaven T, Heidger P, Kreiter C, Duncan J, Dick F (2001) Comparison of a virtual microscope laboratory to a regular microscope laboratory for teaching histology. Anat Rec 265:10-14

11. Harrison JH Jr, Stewart J 3rd (2003) Training in pathology informatics: implementation at the University of Pittsburgh. Arch Pathol Lab Med 127:1019-1025 
12. Heidger PM Jr, Dee F, Consoer D, Leaven T, Duncan J, Kreiter C (2002) Integrated approach to teaching and testing in histology with real and virtual imaging. Anat Rec 269:107-112

13. Helin H, Lundin M, Lundin J, Martikainen P, Tammela T, van der Kwast T, Isola J (2005) Web-based virtual microscopy in teaching and standardizing Gleason grading. Hum Pathol 36:381-386

14. Henricks WH, Boyer PJ, Harrison JH, Tuthill JM, Healy JC (2003) Informatics training in pathology residency programs: proposed learning objectives and skill sets for the new millennium. Arch Pathol Lab Med 127:1009-1018

15. Kumar RK, Velan GM, Korell SO, Kandara M, Dee FR, Wakefield D (2004) Virtual microscopy for learning and assessment in pathology. J Pathol 204:613-618

16. Leong FJ, McGee JO (2001) Automated complete slide digitization: a medium for simultaneous viewing by multiple pathologists. J Pathol 195:508-514

17. Leong FJ, Graham AK, Schwarzmann P, McGee JO (2000) Clinical trial of telepathology as an alternative modality in breast histopathology quality assurance. Telemed J E Health 6:373-377

18. Marchevsky AM, Relan A, Baillie S (2003) Self-instructional "virtual pathology" laboratories using web-based technology enhance medical school teaching of pathology. Hum Pathol 34:423-429

19. Marchevsky AM, Wan Y, Thomas P, Krishnan L, Evans-Simon H, Haber H (2003) Virtual microscopy as a tool for proficiency testing in cytopathology: a model using multiple digital images of Papanicolaou tests. Arch Pathol Lab Med 127:1320-1324
20. Molnar B, Berczi L, Diczhazy C, Tagscherer A, Varga SV, Szende B, Tulassay Z (2003) Digital slide and virtual microscopy based routine and telepathology evaluation of routine gastrointestinal biopsy specimens. J Clin Pathol 56:433-438

21. Okada DH, Binder SW, Felten CL, Strauss JS, Marchevsky AM (1999) "Virtual microscopy" and the internet as telepathology consultation tools: diagnostic accuracy in evaluating melanocytic skin lesions. Am J Dermatopathol 21:525-531

22. Romer DJ, Suster S (2003) Use of virtual microscopy for didactic live-audience presentation in anatomic pathology. Ann Diagn Pathol 7:67-72

23. Romer DJ, Yearsley KH, Ayers LW (2003) Using a modified standard microscope to generate virtual slides. Anat Rec 272B:91-97

24. Steinberg DM, Ali SZ (2001) Application of virtual microscopy in clinical cytopathology. Diagn Cytopathol 25:389-396

25. Weinstein RS, Descour MR, Liang C, Bhattacharyya AK, Graham AR, Davis JR, Scott KM, Richter L, Krupinski EA, Szymus J, Kayser K, Dunn BE (2001) Telepathology overview: from concept to implementation. Hum Pathol 32:1283-1299

26. Weinstein RS, Descour MR, Liang C, Barker G, Scott KM, Richter L, A. KE, Bhattacharyya AK, Davis JR, Graham AR, Rennels M, Russum WC, Goodall JF, Zhou P, Olszak AG, Williams BH, Wyant JC, Bartels PH (2004) An array microscope for ultrarapid virtual slide processing and telepathology. Design, fabrication, and validation Study. Hum Pathol 35:1303-1314

27. Zito FA, Marzullo F, D'Errico D, Salvatore C, Digirolamo R, Labriola A, Pellecchia A (2004) Quicktime virtual reality technology in light microscopy to support medical education in pathology. Mod Pathol 17:728-731 